\title{
Parameter Estimation of Induction Machine at Standstill Using Two-Stage Recursive Least Squares Method
}

\author{
Jinliang Zhang, ${ }^{1,2}$ Longyun Kang, ${ }^{1,2}$ Lingyu Chen, ${ }^{1}$ and Zhihui $\mathrm{Xu}^{3}$ \\ ${ }^{1}$ School of Electric Power, South China University of Technology, Guangzhou, Guangdong 510640, China \\ ${ }^{2}$ Guangdong Key Laboratory of Clean Energy Technology, South China University of Technology, Guangzhou 511458, China \\ ${ }^{3}$ Sunwoda Electronic Corporation Limited, Shenzhen 518108, China
}

Correspondence should be addressed to Jinliang Zhang; boyuyi2108@hotmail.com

Received 13 July 2015; Revised 20 September 2015; Accepted 29 September 2015

Academic Editor: Tarek Ahmed-Ali

Copyright (C) 2015 Jinliang Zhang et al. This is an open access article distributed under the Creative Commons Attribution License, which permits unrestricted use, distribution, and reproduction in any medium, provided the original work is properly cited.

\begin{abstract}
This paper presents a two-stage recursive least squares (TSRLS) algorithm for the electric parameter estimation of the induction machine (IM) at standstill. The basic idea of this novel algorithm is to decouple an identifying system into two subsystems by using decomposition technique and identify the parameters of each subsystem, respectively. The TSRLS is an effective implementation of the recursive least squares (RLS). Compared with the conventional (RLS) algorithm, the TSRLS reduces the number of arithmetic operations. Experimental results verify the effectiveness of the proposed TSRLS algorithm for parameter estimation of IMs.
\end{abstract}

\section{Introduction}

Induction machines (IMs) are widely used in various industrial applications thanks to their particular attractions of simple structure and high reliability [1]. But high-performance control of IM is a persistent and challenging issue with which many researchers are concerned. Among the research results, field-oriented control (FOC) is proved to be a wellestablished control scheme to implement AC drives for IM that can realize high dynamic performance and satisfy rigorous requirements for industrial applications [2, 3]. However, good knowledge of electric parameters is a precondition for the field-oriented controlled IM [4].

Traditional methods to obtain the IM electric parameters known as locked-rotor and no-load tests have problems of insufficient accuracy to apply in high-performance drive and limited experimental conditions [5]. Therefore, a variety of new IM parameter estimation techniques have emerged in recent years. According to the operating conditions of the IM while performing the parameter estimation, they can be classified into "online" and "offline" estimations $[6,7]$. Although online estimation can be adaptive for the variation of parameters, the fraught global stability and the considerable computational burden deteriorate its applications.
By contrast, offline estimation is a simpler choice and can act as an indispensable guarantee for the start-up of the drive. Moreover, the result values from offline estimation can be a good initialization for performing an online estimation [6].

In $[5,6]$, "self-commissioning" is introduced which indicates a present trend of performing an offline estimation at standstill without any extra hardware and making the control system operate automatically after the drive installation. Such offline estimation methods have been discussed in many literatures. The authors in [6] designed a parallel adaptive observer with excellent noise rejection, which is used to give a recursive estimate of the magnetic flux against magnetic saturation and incorrect estimation of the magnetic parameters. In [7], a method based on three frequency-domain tests is adopted, with a phase-sensitive detection technique used for noise immunity and measurement accuracy. In [8], the authors present a current injection identification method that utilizes the general frequency characteristics of the rotor bar to track the parameters by injecting two exciting currents with different frequencies and employing closed-loop current control, despite leaving the problem of inductance saturation unsolved. Furthermore, the authors of [9] propose an automatic procedure for the complete identification of the inverse- $\Gamma$ equivalent circuit for induction motors at standstill, 
which takes into account both the magnetic nonlinearity and compensation of the inverter nonideality. Its step-bystep approach makes use of the voltage inverter as a precise voltage probe and avoids any direct voltage measurement. The offline self-commissioning procedure for the automatic IM parameter estimation in [10] also consists of a step-by-step approach with several different test signals in sequence and is capable of mapping both inverter and motor parameters nonlinearities. And in [5], the RLS algorithm is applied to estimate IM parameters based on continuous-time model at standstill, and specifically, a vector constructing method is used to cancel the normally indispensable analog or digital differentiators.

Among the self-commissioning offline estimation methods, the recursive least squares (RLS) algorithm is a prominent and widespread-used method that has advantages of high identifying accuracy and compatibility for both online and offline estimations [5, 11-15]. The RLS-based algorithms described in $[5,11-15]$ have good performance, but the applicability of performing these algorithms in real-time is generally limited by the complex mathematical operations; only high-performance microcontroller can qualify for this work.

To reduce the computational complexity of the RLS, this paper presents a two-stage recursive least squares (TSRLS) algorithm for electric parameter estimation of the induction machine at standstill. The basic idea is to decompose an identifying IM model into two parallel subsystems and identify each subsystem, respectively. The proposed algorithm is an effective implementation of recursive least squares algorithm. Compared to the conventional RLS, the TSRLS can reduce the computational burden. To facilitate the understanding, the complete equations of this algorithm are presented and compared to a straight implementation of the conventional RLS equations.

The paper is organized as follows. In Section 2, the RLS estimation model of the IM at standstill is introduced. In Section 3, according to the discrete RLS estimation model of the IM, the TSRLS algorithm for electric parameter estimation of the IM at standstill is developed by the decomposition approach. For comparison, the conventional RLS estimation algorithm is given in Section 4. In Section 5, experimental results are discussed. Finally, a conclusion wraps up the paper.

\section{Induction Machine Model at Standstill}

2.1. The Dynamic Model of the IM. As elaborated in [15], the dynamic mathematical model of an IM in the stationary $(\alpha \beta)$ reference frame is as follows:

$$
\begin{aligned}
& \frac{d \psi_{r \alpha}}{d t}=\frac{L_{m}}{T_{r}} i_{s \alpha}-\frac{1}{T_{r}} \psi_{r \alpha}-p \omega_{r} \psi_{r \beta}, \\
& \frac{d \psi_{r \beta}}{d t}=\frac{L_{m}}{T_{r}} i_{s \beta}-\frac{1}{T_{r}} \psi_{r \beta}+p \omega_{r} \psi_{r \alpha}, \\
& \frac{d i_{s \alpha}}{d t}=-\gamma i_{s \alpha}+\frac{L_{m}}{\sigma L_{s} L_{r} T_{r}} \psi_{r \alpha}+\frac{L_{m}}{\sigma L_{s} L_{r}} p \omega_{r} \psi_{r \beta}
\end{aligned}
$$

$$
\begin{gathered}
+\frac{1}{\sigma L_{s}} u_{s \alpha}, \\
\frac{d i_{s \beta}}{d t}=-\gamma i_{s \beta}+\frac{L_{m}}{\sigma L_{s} L_{r} T_{r}} \psi_{r \beta}-\frac{L_{m}}{\sigma L_{s} L_{r}} p \omega_{r} \psi_{r \alpha} \\
+\frac{1}{\sigma L_{s}} u_{s \beta}, \\
T_{e}=\frac{p L_{m}}{L_{r}}\left(\psi_{r \alpha} i_{s \beta}-\psi_{r \beta} i_{s \alpha}\right),
\end{gathered}
$$

where $\left(i_{s \alpha}, i_{s \beta}\right),\left(u_{s \alpha}, u_{s \beta}\right)$, and $\left(\psi_{r \alpha}, \psi_{r \beta}\right)$ are the stator current, stator voltage, and rotor flux in the stationary reference frame. $L_{s}, L_{r}$, and $L_{m}$ are the stator inductance, rotor inductance, and mutual inductance, respectively, and $R_{r}$ and $R_{s}$ are the rotor resistance and stator resistance. $T_{e}$ is the electromagnetic torque produced by the IM. The rotor angular velocity $\omega_{r}$ is measured in mechanical radians per second and $p$ is the number of pole pairs. Furthermore, $T_{r}=L r / R_{r}$ is the rotor time constant and other parameters used in (1) are defined as $\sigma=1-L_{m}^{2} / L_{r} L_{s}$ and $\gamma=R_{s} / \sigma L_{s}+R_{r} L_{m}^{2} / \sigma L_{s} L_{r}^{2}$, respectively.

Assuming that the IM is at standstill, the machine is controlled to produce zero electromagnetic torque with $\omega_{r}=$ 0 . The electromagnetic torque expression in (1) shows that if only one phase of the equivalent machine model is excited by the stator voltage, then the produced electromagnetic torque is null. Since the $\alpha$-axis and $\beta$-axis components of the stator voltage have the same expressing form, the following relation can be derived from (1) using only $\alpha$-axis components and $u_{s \beta}, \psi_{r \beta}$, and $i_{s \beta}$ are all set to zero:

$$
\begin{aligned}
& \frac{d \psi_{r \alpha}}{d t}=\frac{L_{m}}{T_{r}} i_{s \alpha}-\frac{1}{T_{r}} \psi_{r \alpha}, \\
& \frac{d i_{s \alpha}}{d t}=-\gamma i_{s \alpha}+\frac{L_{m}}{\sigma L_{s} L_{r} T_{r}} \psi_{r \alpha}+\frac{1}{\sigma L_{s}} u_{s \alpha} .
\end{aligned}
$$

Performing Laplace transform to (2), we have

$$
\begin{aligned}
\left(T_{r} s+1\right) \psi_{r \alpha}(s) & =L_{m} i_{s \alpha}(s), \\
(s+\gamma) i_{s \alpha}(s)+\frac{L_{m}}{\sigma L_{s} L_{r} T_{r}} \psi_{r \alpha}(s) & =\frac{1}{\sigma L_{s}} u_{s \alpha}(s) .
\end{aligned}
$$

Substituting (3) into (4), the following input-output relation can be acquired in the Laplace domain:

$$
H(s)=\frac{i_{s \alpha}}{u_{s \alpha}}=\frac{b_{1} s+b_{0}}{s^{2}+a_{1} s+a_{0}},
$$

where

$$
\begin{aligned}
& b_{1}=\frac{1}{\sigma L_{s}}, \\
& b_{0}=\frac{1}{\sigma L_{s} T_{r}},
\end{aligned}
$$




$$
\begin{aligned}
& a_{1}=\frac{R_{s}}{\sigma L_{s}}+\frac{1}{\sigma T_{r}}, \\
& a_{0}=\frac{R_{s}}{\sigma L_{s} T_{r}} .
\end{aligned}
$$

2.2. The RLS Estimation Model. In order to use the RLS method, (5) should be rewritten into a linear regression equation as follows:

$$
y(t)=\varphi^{T}(t) \theta
$$

where $y(t), \varphi(t)$, and $\theta$ are the prediction vector, measured signal vector, and parametric vector, respectively. Since (5) is a second-order system, it is necessary to define a transformation second-order filter $\Gamma(s)=\left(s+h_{0}\right)\left(s+h_{1}\right)$. Define $Z=s^{2} i_{s \alpha} / \Gamma(s)$, and then the following relations are obtained using (5):

$$
\begin{aligned}
Z & =\frac{s^{2} i_{s \alpha}}{\Gamma(s)}=\frac{b_{1} s u_{s \alpha}+b_{0} u_{s \alpha}-a_{1} s i_{s \alpha}-a_{0} i_{s \alpha}}{\Gamma(s)}, \\
Z & =\frac{s^{2} i_{s \alpha}}{\Gamma(s)}=\frac{\Gamma(s) i_{s \alpha}-\left(\left(h_{1}+h_{0}\right) s+h_{1} h_{0}\right) i_{s \alpha}}{\Gamma(s)} \\
& =i_{s \alpha}-\frac{\left(\left(h_{1}+h_{0}\right) s+h_{1} h_{0}\right) i_{s \alpha}}{\Gamma(s)} .
\end{aligned}
$$

From (9) we have

$$
i_{s \alpha}=\frac{\left(\left(h_{1}+h_{0}\right) s+h_{1} h_{0}\right) i_{s \alpha}}{\Gamma(s)}+Z .
$$

Then

$$
\begin{aligned}
i_{s \alpha}= & \frac{\left(\left(h_{1}+h_{0}-a_{1}\right) s+\left(h_{1} h_{0}-a_{0}\right)\right) i_{s \alpha}}{\Gamma(s)} \\
& +\frac{b_{1} s+b_{0}}{\Gamma(s)} u_{s \alpha} .
\end{aligned}
$$

The three components of the linear regression equation (7) can be expressed based on the above equations as

$$
\begin{aligned}
& y(t)=i_{s \alpha}, \\
& \theta=\left[\begin{array}{c}
\theta_{a} \\
\theta_{b}
\end{array}\right]=\left[\begin{array}{c}
\theta_{1} \\
\theta_{2} \\
\theta_{3} \\
\theta_{4}
\end{array}\right]=\left[\begin{array}{c}
\frac{b_{0}-b_{1} h_{1}}{h_{0}-h_{1}} \\
\frac{b_{1} h_{0}-b_{0}}{h_{0}-h_{1}} \\
\frac{a_{1} h_{1}-a_{0}-h_{1}^{2}}{h_{0}-h_{1}} \\
\frac{-a_{1} h_{0}+a_{0}+h_{0}^{2}}{h_{0}-h_{1}}
\end{array}\right], \\
& \varphi(t)=\left[\begin{array}{ll}
\varphi_{a}(t) & \varphi_{b}(t)
\end{array}\right]^{T}=\left[\begin{array}{llll}
d_{1} & d_{2} & d_{3} & d_{4}
\end{array}\right]^{T} \\
& =\left[\begin{array}{llll}
\frac{u_{s \alpha}}{s+h_{1}} & \frac{u_{s \alpha}}{s+h_{0}} & \frac{i_{s \alpha}}{s+h_{1}} & \frac{i_{s \alpha}}{s+h_{0}}
\end{array}\right]^{T},
\end{aligned}
$$

where

$$
\begin{aligned}
\theta_{a} & =\left[\begin{array}{ll}
\theta_{1} & \theta_{2}
\end{array}\right]^{T}, \\
\theta_{b} & =\left[\begin{array}{ll}
\theta_{3} & \theta_{4}
\end{array}\right]^{T}, \\
\varphi_{a}(t) & =\left[\begin{array}{ll}
\frac{u_{s \alpha}}{s+h_{1}} & \frac{u_{s \alpha}}{s+h_{0}}
\end{array}\right]^{T}, \\
\varphi_{b}(t) & =\left[\begin{array}{ll}
\frac{i_{s \alpha}}{s+h_{1}} & \frac{i_{s \alpha}}{s+h_{0}}
\end{array}\right]^{T} .
\end{aligned}
$$

Note that the measured signal vector in (14) is of only firstorder instead of second-order because a second-order filter $\Gamma(s)$ is used in advance.

Discretion of the RLS estimation model is required for its digital implementation, so (7) is turned into discrete form as

$$
y(k)=\varphi^{T}(k) \theta
$$

during a sampling period $T$. The corresponding discrete form of (14) can be obtained by performing the bilinear $z$ transform, that is, replacing the Laplace operators by the equation $s=2\left(1+z^{-1}\right) / T\left(1+z^{-1}\right)$, and we obtain the following recursive equation:

$$
\begin{aligned}
\varphi(k) & =\left[\begin{array}{l}
d_{1}(k) \\
d_{2}(k) \\
d_{3}(k) \\
d_{3}(k)
\end{array}\right] \\
= & {\left[\begin{array}{l}
c_{0} d_{1}(k-1)+c_{1} u_{s \alpha}(k)+c_{2} u_{s \alpha}(k-1) \\
c_{3} d_{2}(k-1)+c_{4} u_{s \alpha}(k)+c_{5} u_{s \alpha}(k-1) \\
c_{0} d_{3}(k-1)+c_{1} i_{s \alpha}(k)+c_{2} i_{s \alpha}(k-1) \\
c_{3} d_{4}(k-1)+c_{4} i_{s \alpha}(k)+c_{5} i_{s \alpha}(k-1)
\end{array}\right], }
\end{aligned}
$$

where

$$
\begin{aligned}
& c_{0}=\frac{2-h_{1} T}{2+h_{1} T}, \\
& c_{1}=c_{2}=\frac{T}{2+T h_{1}}, \\
& c_{3}=\frac{2-h_{0} T}{2+h_{0} T}, \\
& c_{5}=c_{4}=\frac{T}{2+T h_{0}} .
\end{aligned}
$$

Supposing that $\hat{\theta}$ is the estimated value of $\theta$, the following parameters of the IM can be retrieved from (6) and (13):

$$
\begin{aligned}
& \widehat{R}_{s}=\frac{\widehat{a}_{0}}{\widehat{b}_{0}}=\frac{h_{1} h_{0}-h_{1} \widehat{\theta}_{4}-h_{0} \widehat{\theta}_{3}}{h_{0} \widehat{\theta}_{1}+h_{1} \widehat{\theta}_{2}}, \\
& \widehat{R}_{r}=\frac{\widehat{a}_{1}}{\widehat{b}_{1}}-\widehat{R}_{s}=\frac{h_{1}+h_{0}-\widehat{\theta}_{3}-\widehat{\theta}_{4}}{\widehat{\theta}_{1}+\widehat{\theta}_{2}}-\widehat{R}_{s},
\end{aligned}
$$




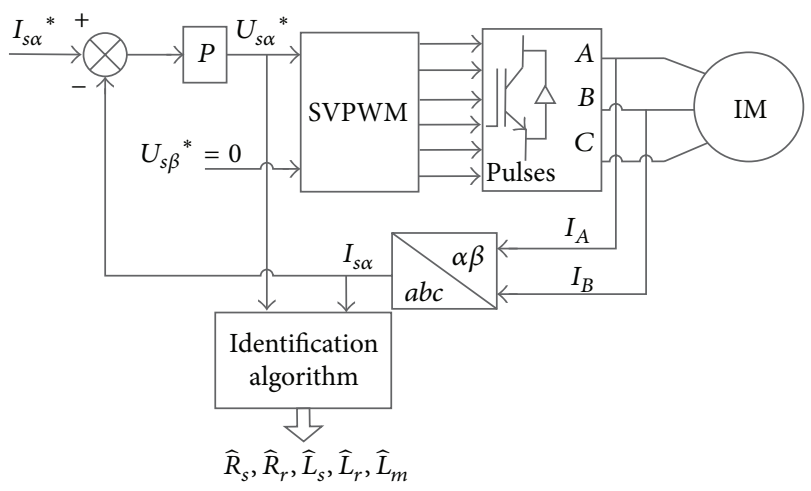

FIGURE 1: Schematic of the implementation of the IM parameter estimation at standstill.

$$
\begin{gathered}
\widehat{L}_{s}=\widehat{L}_{r}=\frac{\widehat{b}_{1} \widehat{R}_{r}}{\widehat{b}_{0}}=\frac{\left(\widehat{\theta}_{1}+\widehat{\theta}_{2}\right) \widehat{R}_{r}}{h_{0} \widehat{\theta}_{1}+h_{1} \widehat{\theta}_{2}}, \\
\widehat{L}_{m}=\sqrt{\widehat{L}_{s}^{2}-\frac{\widehat{L}_{s}}{\widehat{b}_{1}}}=\sqrt{\widehat{L}_{s}^{2}-\frac{\widehat{L}_{s}}{\left(\widehat{\theta}_{1}+\widehat{\theta}_{2}\right)}} .
\end{gathered}
$$

The schematic of the implementation of the IM parameter estimation is shown in Figure 1. The reference $\alpha$-axis component of the stator current $I_{s \alpha}{ }^{*}$ consists of two sinusoidal signals with distinct frequencies. The reference $\beta$-axis component of the stator voltage $U_{s \beta}{ }^{*}$ is set to zero. Therefore, there will be no electromagnetic torque generated by the IM.

\section{Two-Stage Recursive Least Squares Algorithm}

The basic idea of the TSRLS algorithm is to decouple the system into two subsystems (i.e., decouple the parameter vector and the measured signal vector into two subvectors, resp.) and then identify the parameters of each subsystem utilizing the RLS estimation. This algorithm can effectively save computational cost compared to the conventional RLS. Considering the noise and parameter errors, the discrete linear regression equation of the system is described as

$$
y(k)=\varphi^{T}(k) \theta+w(k)
$$

where

$$
\begin{aligned}
y(k) & =i_{s \alpha}(k), \\
\theta & =\left[\begin{array}{ll}
\theta_{a} & \theta_{b}
\end{array}\right]^{T}, \\
\varphi(k) & =\left[\begin{array}{ll}
\varphi_{a}(k) & \varphi_{b}(k)
\end{array}\right]^{T}, \\
\theta_{a} & =\left[\begin{array}{l}
\theta_{1} \\
\theta_{2}
\end{array}\right]=\left[\begin{array}{c}
\frac{b_{0}-b_{1} h_{1}}{h_{0}-h_{1}} \\
\frac{b_{1} h_{0}-b_{0}}{h_{0}-h_{1}}
\end{array}\right],
\end{aligned}
$$

$$
\begin{gathered}
\theta_{b}=\left[\begin{array}{l}
\theta_{3} \\
\theta_{4}
\end{array}\right]=\left[\begin{array}{c}
\frac{a_{1} h_{1}-a_{0}-h_{1}^{2}}{h_{0}-h_{1}} \\
\frac{-a_{1} h_{0}+a_{0}+h_{0}^{2}}{h_{0}-h_{1}}
\end{array}\right], \\
\varphi_{a}(k)=\left[\begin{array}{l}
d_{1}(k) \\
d_{2}(k)
\end{array}\right]^{T}, \\
\varphi_{b}(k)=\left[\begin{array}{l}
d_{3}(k) \\
d_{4}(k)
\end{array}\right]^{T},
\end{gathered}
$$

where $w(k)$ is a zero-mean white noise sequence with covariance matrix $Q=\sigma^{2} \cdot I_{N \times N}$. The TSRLS algorithm can be obtained by using decomposition method, so it is necessary to define two intermediate variables:

$$
\begin{aligned}
& y_{1}(k)=y(k)-\varphi_{b}^{T}(k) \theta_{b}, \\
& y_{2}(k)=y(k)-\varphi_{a}^{T}(k) \theta_{a} .
\end{aligned}
$$

Then, the system in (20) can be decoupled into the following two fictitious subsystems:

$$
\begin{aligned}
& y_{1}(k)=\varphi_{a}^{T}(k) \theta_{a}+w(k), \\
& y_{2}(k)=\varphi_{b}{ }^{T}(k) \theta_{b}+w(k) .
\end{aligned}
$$

These two subsystems contain the parameter vectors $\theta_{a}$ and $\theta_{b}$ that need to be identified. Consider the data from $k=1$ to $k=N(N \gg 4)$ and define the stacked output vectors $Y(N), Y_{1}(N)$, and $Y_{2}(N)$, the stacked measured signal vectors $\Phi_{a}(N), \Phi_{b}(N)$, and the stacked white noise vector $W(N)$ as

$$
Y(N)=\left[\begin{array}{c}
y(1) \\
y(2) \\
\vdots \\
y(N)
\end{array}\right] \in \mathbb{R}^{N}
$$

$$
W(N)=\left[\begin{array}{c}
w(1) \\
w(2) \\
\vdots \\
w(N)
\end{array}\right] \in \mathbb{R}^{N},
$$$$
\Phi_{a}(N)=\left[\begin{array}{c}
\varphi_{a}{ }^{T}(1) \\
\varphi_{a}{ }^{T}(2) \\
\vdots \\
\varphi_{a}{ }^{T}(N)
\end{array}\right] \in \mathbb{R}^{N \times 2},
$$ 


$$
\begin{aligned}
& \Phi_{b}(N)=\left[\begin{array}{c}
\varphi_{b}{ }^{T}(1) \\
\varphi_{b}{ }^{T}(2) \\
\vdots \\
\varphi_{b}{ }^{T}(N)
\end{array}\right] \in \mathbb{R}^{N \times 2}, \\
& Y_{1}(N)=\left[\begin{array}{c}
y_{1}(1) \\
y_{1}(2) \\
\vdots \\
y_{1}(N)
\end{array}\right] \in \mathbb{R}^{N}, \\
& \Phi_{a}{ }^{T}(N) \Phi_{a}(N) \widehat{\theta}_{a}(N) \\
& =\Phi_{a}{ }^{T}(N)\left[Y(N)-\Phi_{b}(N) \theta_{b}\right], \\
& \Phi_{b}{ }^{T}(N) \Phi_{b}(N) \widehat{\theta}_{b}(N) \\
& =\Phi_{b}{ }^{T}(N)\left[Y(N)-\Phi_{a}(N) \theta_{a}\right] \text {. }
\end{aligned}
$$$$
Y_{2}(N)=\left[\begin{array}{c}
y_{2}(1) \\
y_{2}(2) \\
\vdots \\
y_{2}(N)
\end{array}\right] \in \mathbb{R}^{N} \text {. }
$$

The matrices $\left[\Phi_{a}{ }^{T}(N) \Phi_{a}(N)\right]$ and $\left[\Phi_{b}{ }^{T}(N) \Phi_{b}(N)\right]$ are nonsingular, because the measured signal vectors $\varphi_{a}(t)$ and $\varphi_{b}(t)$ are persistently exciting in the estimation system. From the above two equations, we have the following least squares estimations of $\theta_{a}$ and $\theta_{b}$ at iteration $N$ :

$$
\begin{gathered}
\widehat{\theta}_{a}(N)=\left[\Phi_{a}^{T}(N) \Phi_{a}(N)\right]^{-1} \Phi_{a}^{T}(N) \\
\cdot\left[Y(N)-\Phi_{b}(N) \theta_{b}\right], \\
\widehat{\theta}_{b}(N)=\left[\Phi_{b}^{T}(N) \Phi_{b}(N)\right]^{-1} \Phi_{b}^{T}(N) \\
\cdot\left[Y(N)-\Phi_{a}(N) \theta_{a}\right] .
\end{gathered}
$$

To avoid computing the matrix inversion and reduce the computational complexity, we define two covariance matrices:

$$
\begin{aligned}
& P_{a}(N)=\left[\Phi_{a}^{T}(N) \Phi_{a}(N)\right]^{-1}, \\
& P_{b}(N)=\left[\Phi_{b}{ }^{T}(N) \Phi_{b}(N)\right]^{-1} .
\end{aligned}
$$

It follows that

$$
\begin{aligned}
& J_{1}\left(\theta_{a}\right)=\left\|Y_{1}(N)-\Phi_{a}(N) \theta_{a}\right\|^{2}, \\
& J_{2}\left(\theta_{b}\right)=\left\|Y_{2}(N)-\Phi_{b}(N) \theta_{b}\right\|^{2} .
\end{aligned}
$$

For these two optimization problems, let the partial derivatives of $J_{1}\left(\theta_{a}\right)$ and $J_{2}\left(\theta_{b}\right)$ with respect to $\theta_{a}$ and $\theta_{b}$ be zero, respectively; namely,

$$
\begin{aligned}
& \frac{\partial J_{1}\left(\theta_{a}\right)}{\partial \theta_{a}}=-2 \Phi_{a}^{T}(N)\left[Y_{1}(N)-\Phi_{a}(N) \theta_{a}\right] \\
& \quad=-2 \Phi_{a}^{T}(N)\left[Y(N)-\Phi_{b}(N) \theta_{b}-\Phi_{a}(N) \theta_{a}\right] \\
& \quad=0, \\
& \frac{\partial J_{2}\left(\theta_{b}\right)}{\partial \theta_{b}}=-2 \Phi_{b}^{T}(N)\left[Y_{2}(N)-\Phi_{b}(N) \theta_{b}\right] \\
& \quad=-2 \Phi_{b}{ }^{T}(N)\left[Y(N)-\Phi_{a}(N) \theta_{a}-\Phi_{b}(N) \theta_{b}\right] \\
& =0 .
\end{aligned}
$$

$$
\begin{aligned}
& P_{a}^{-1}(N+1)=P_{a}^{-1}(N)+\varphi_{a}(N+1) \varphi_{a}{ }^{T}(N+1), \\
& P_{b}^{-1}(N+1)=P_{b}^{-1}(N)+\varphi_{b}(N+1) \varphi_{b}{ }^{T}(N+1) .
\end{aligned}
$$

In terms of (31), (33), (35), and the definitions of $Y(N)$, $\Phi_{a}(N)$, and $\Phi_{b}(N)$, we get

$$
\begin{aligned}
& \widehat{\theta}_{a}(N+1)=P_{a}(N+1) \Phi_{a}^{T}(N+1)[Y(N+1) \\
& \left.\quad-\Phi_{b}(N+1) \theta_{b}\right]=P_{a}(N+1) \\
& \cdot\left\{\Phi_{a}^{T}(N)\left[Y(N)-\Phi_{b}(N) \theta_{b}\right]\right. \\
& \left.\quad+\varphi_{a}(N+1)\left[y(N+1)-\varphi_{b}^{T}(N+1) \theta_{b}\right]\right\} \\
& \quad=P_{a}(N+1) \\
& \quad \cdot\left\{P_{a}^{-1}(N) P_{a}(N) \Phi_{a}{ }^{T}(N)\left[Y(N)-\Phi_{b}(N) \theta_{b}\right]\right. \\
& \left.\quad+\varphi_{a}(N+1)\left[y(N+1)-\varphi_{b}^{T}(N+1) \theta_{b}\right]\right\}
\end{aligned}
$$




$$
\begin{aligned}
& =P_{a}(N+1)\left[P_{a}^{-1}(N+1)\right. \\
& \left.-\varphi_{a}(N+1) \varphi_{a}^{T}(N+1)\right] \widehat{\theta}_{a}(N)+P_{a}(N+1) \\
& \cdot \varphi_{a}(N+1)\left[y(N+1)-\varphi_{b}^{T}(N+1) \theta_{b}\right] \\
& =\hat{\theta}_{a}(N)+P_{a}(N+1) \varphi_{a}(N+1)[y(N+1) \\
& \left.-\varphi_{b}{ }^{T}(N+1) \theta_{b}-\varphi_{a}{ }^{T}(N+1) \hat{\theta}_{a}(N)\right] .
\end{aligned}
$$

Analogously, the following relation is obtained from (32), (34), and (36):

$$
\begin{aligned}
\widehat{\theta}_{b}(N+1)=\widehat{\theta}_{b}(N)+P_{b}(N+1) \varphi_{b}(N+1) \\
\cdot[y(N+1) \\
\left.-\varphi_{a}{ }^{T}(N+1) \theta_{a}-\varphi_{b}{ }^{T}(N+1) \hat{\theta}_{b}(N)\right] .
\end{aligned}
$$

Applying the matrix inversion formula

$$
[A+B C]^{-1}=A^{-1}-A^{-1} B\left[I+C A^{-1} B\right]^{-1} C A^{-1}
$$

to (33) and (34) yields

$$
\begin{aligned}
& P_{a}(N+1) \\
& \quad=P_{a}(N)-\frac{P_{a}(N) \varphi_{a}(N+1) \varphi_{a}{ }^{T}(N+1) P_{a}(N)}{1+\varphi_{a}^{T}(N+1) P_{a}(N) \varphi_{a}(N+1)}, \\
& P_{b}(N+1) \\
& \quad=P_{b}(N)-\frac{P_{b}(N) \varphi_{b}(N+1) \varphi_{b}^{T}(N+1) P_{b}(N)}{1+\varphi_{b}^{T}(N+1) P_{b}(N) \varphi_{b}(N+1)} .
\end{aligned}
$$

Substituting (40) and (41) into the right side of (37) and (38), the following recursive least squares algorithms are obtained:

$$
\begin{aligned}
& \hat{\theta}_{a}(N+1)=\hat{\theta}_{a}(N)+G_{a}(N+1) \\
& \cdot\left[y(N+1)-\varphi_{b}(N+1) \theta_{b}-\varphi_{a}(N+1) \hat{\theta}_{a}(N)\right], \\
& G_{a}(N+1)=P_{a}(N) \varphi_{a}^{T}(N+1) \\
& \quad \cdot\left(1+\varphi_{a}(N+1) P_{a}(N) \varphi_{a}^{T}(N+1)\right)^{-1}, \\
& P_{a}(N+1)=P_{a}(N)-G_{a}(N+1) \varphi_{a}(N+1) P_{a}(N) \\
& \quad P_{a}(0)=\sigma^{2} I,
\end{aligned}
$$

$$
\begin{aligned}
& \hat{\theta}_{b}(N+1)=\hat{\theta}_{b}(N)+G_{b}(N+1) \\
& \cdot\left[y(N+1)-\varphi_{a}(N+1) \theta_{a}-\varphi_{b}(N+1) \widehat{\theta}_{b}(N)\right], \\
& G_{b}(N+1)=P_{b}(N) \varphi_{b}^{T}(N+1) \\
& \quad \cdot\left(1+\varphi_{b}(N+1) P_{b}(N) \varphi_{b}^{T}(N+1)\right)^{-1}, \\
& P_{b}(N+1)=P_{b}(N)-G_{b}(N+1) \varphi_{b}(N+1) P_{b}(N) \\
& \quad P_{b}(0)=\sigma^{2} I .
\end{aligned}
$$

However, due to the unknown parameter vectors $\theta_{b}$ and $\theta_{a}$ in the right sides of (42) and (45), it is difficult to compute their estimations $\hat{\theta}_{a}$ and $\hat{\theta}_{b}$. The solution is to replace the unknown $\theta_{b}$ and $\theta_{a}$ with their preceding estimations $\widehat{\theta}_{b}(N)$ and $\hat{\theta}_{a}(N)$, respectively; then (42) and (45) can be written as

$$
\begin{array}{r}
\widehat{\theta}_{a}(N+1)=\hat{\theta}_{a}(N)+G_{a}(N+1)[y(N+1) \\
\left.-\varphi_{b}(N+1) \hat{\theta}_{b}(N)-\varphi_{a}(N+1) \hat{\theta}_{a}(N)\right], \\
\widehat{\theta}_{b}(N+1)=\widehat{\theta}_{b}(N)+G_{b}(N+1)[y(N+1) \\
\left.-\varphi_{a}(N+1) \hat{\theta}_{a}(N)-\varphi_{b}(N+1) \hat{\theta}_{b}(N)\right] .
\end{array}
$$

Based on the above analysis, the TSRLS algorithm can be organized by the next two parts $[16,17]$. The first part of the TSRLS for parametric vector $\theta_{a}$ estimation is

$$
\begin{aligned}
& \widehat{\theta}_{a}(N+1)=\hat{\theta}_{a}(N)+G_{a}(N+1)[y(N+1) \\
& \left.\quad-\varphi_{b}(N+1) \hat{\theta}_{b}(N)-\varphi_{a}(N+1) \hat{\theta}_{a}(N)\right] \\
& \quad=\widehat{\theta}_{a}(N)+G_{a}(N+1)[y(N+1) \\
& \quad-\varphi(N+1) \hat{\theta}(N)] \widehat{\theta}_{a}(0)=0, \\
& G_{a}(N+1)=P_{a}(N) \varphi_{a}{ }^{T}(N+1)(1 \\
& \left.\quad+\varphi_{a}(N+1) P_{a}(N) \varphi_{a}^{T}(N+1)\right)^{-1}, \\
& P_{a}(N+1)=P_{a}(N)-G_{a}(N+1) \varphi_{a}(N+1) P_{a}(N) \\
& \varphi_{a}(N+1)=\left[d_{1}(N+1) d_{2}(N+1)\right]^{T} .
\end{aligned}
$$

The second part for estimating the parametric vector $\theta_{b}$ is

$$
\begin{aligned}
\widehat{\theta}_{b} & (N+1)=\widehat{\theta}_{b}(N)+G_{b}(N+1)[y(N+1) \\
& \left.-\varphi_{a}(N+1) \hat{\theta}_{a}(N)-\varphi_{b}(N+1) \hat{\theta}_{b}(N)\right] \\
& =\widehat{\theta}_{b}(N)+G_{b}(N+1)[y(N+1) \\
& -\varphi(N+1) \hat{\theta}(N)] \widehat{\theta}_{b}(0)=0
\end{aligned}
$$




$$
\begin{aligned}
& G_{b}(N+1)=P_{b}(N) \varphi_{b}^{T}(N+1)(1 \\
& \left.\quad+\varphi_{b}(N+1) P_{b}(N) \varphi_{b}^{T}(N+1)\right)^{-1}, \\
& P_{b}(N+1)=P_{b}(N)-G_{b}(N+1) \varphi_{b}(N+1) P_{b}(N) \\
& \quad P_{b}(0)=\sigma^{2} I, \\
& \varphi_{b}(N+1)=\left[\begin{array}{lll}
d_{3}(N+1) & d_{4}(N+1)
\end{array}\right]^{T},
\end{aligned}
$$

where $G_{a}(N+1)$ and $G_{b}(N+1)$ are two gain vectors and $P_{a}(N)$ and $P_{b}(N)$ are two covariance matrices.

The steps involved in the TSRLS algorithm in (49)-(56) to estimate the electric parameters of the IM are listed below.

(1) Set the initial values $P_{a}(0)=\sigma^{2} I, P_{b}(0)=\sigma^{2} I, \widehat{\theta}_{a}(0)=$ $0, \widehat{\theta}_{b}(0)=0, u_{s \alpha}(0)=0, i_{s \alpha}(0)=0, \sigma^{2}=9 \times 10^{6}$.

(2) Collect the input/output data $u_{s \alpha}(N+1)$ and $i_{s \alpha}(N+1)$, from $\varphi_{a}(N+1)$ by (52) and $\varphi_{b}(N+1)$ by (56).

(3) Compute $G_{a}(N+1)$ by (50), $P_{a}(N+1)$ by (51), $G_{b}(N+$ $1)$ by (54), and $P_{b}(N+1)$ by (55).

(4) Update the parameter estimations $\widehat{\theta}_{a}(N+1)$ and $\widehat{\theta}_{b}(N+1)$ by (49) and (53), respectively.

(5) Compute the electric parameter estimations of the IM by (16).

(6) Increase $N$ by 1 and go to step (2), and continue the recursive calculation.

The flowchart of computing the electric parameter estimations $\widehat{R}_{s}, \widehat{R}_{r}, \widehat{L}_{s}, \widehat{L}_{r}$, and $\widehat{L}_{m}$ is shown in Figure 2 .

\section{Conventional Recursive Least Squares Algorithm}

To compare with the proposed TSRLS algorithm, the conventional RLS algorithm for parameter estimation of the IM is introduced in this section briefly. Treating $\varphi(k)$ as the measured signal vector and $\theta$ as the parametric vector, $y(k)$ is chosen as the prediction vector; minimizing the criterion function

$$
J(\theta)=\sum_{k=1}^{N}\left[y(k)-\varphi^{T}(k) \theta\right]^{2}
$$

leads to the following conventional recursive least squares algorithm for estimating vector $\theta$ :

$$
\begin{aligned}
& \widehat{\theta}(N+1)=\widehat{\theta}(N)+G(N+1) \\
& \cdot[y(N+1)-\varphi(N+1) \widehat{\theta}(N)] \widehat{\theta}(0)=0, \\
& G(N+1)=P(N) \varphi^{T}(N+1) \\
& \cdot\left(1+\varphi(N+1) P(N) \varphi^{T}(N+1)\right)^{-1},
\end{aligned}
$$

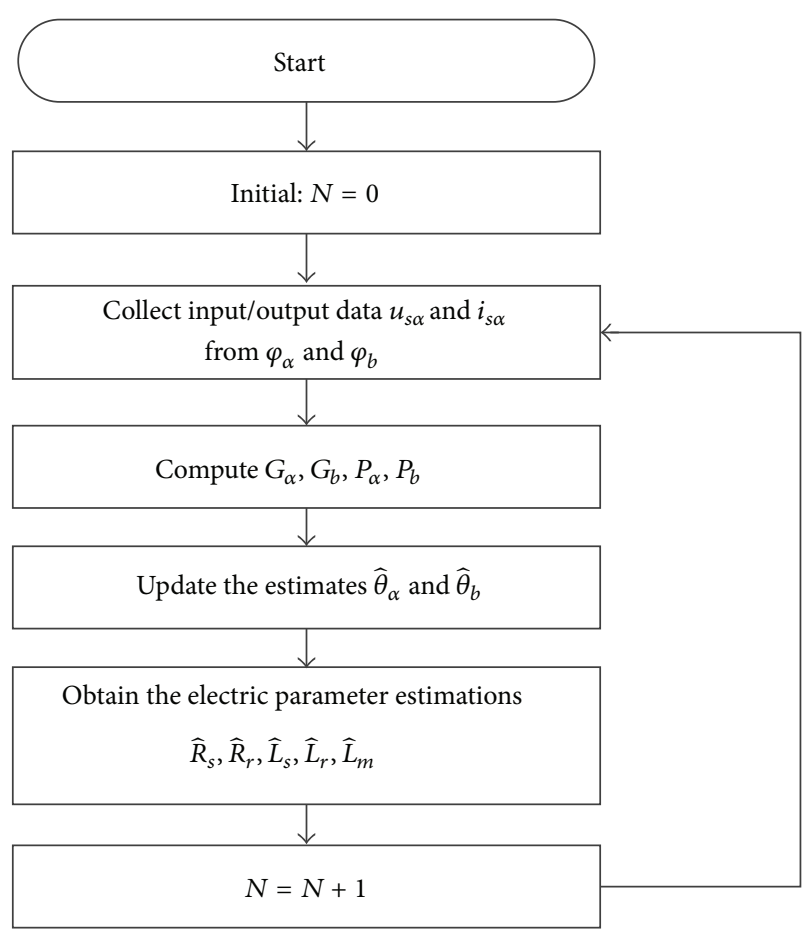

FIGURE 2: Flowchart for computing the electric parameter estimations using the TSRLS.

$$
\begin{aligned}
& P(N+1)=P(N)-G(N+1) \varphi(N+1) P(N) \\
& \\
& P(0)=\sigma^{2} I, \\
& \varphi(N+1) \\
& \quad=\left[\begin{array}{llll}
d_{1}(N+1) & d_{2}(N+1) & d_{3}(N+1) & d_{4}(N+1)
\end{array}\right]^{T},
\end{aligned}
$$

where $G(N+1)$ and $P(N)$ are the gain vectors and the covariance matrices, respectively.

\section{Experimental Results}

The experiments of this paper aim at making a comparison between the TSRLS and the RLS and verifying the validity and feasibility of the TSRLS parameter estimation algorithm for the IM. The schematic of the proposed TSRLS parameter estimation method is shown in Figure 1. The overall experimental setup is shown in Figure 3 and the "real" electric parameters of the IM, which are calculated from traditional no-load and locked-rotor test, are listed in Table 1. The experimental hardware consists of an Expert3 control system from Myway Company and a three-phase, two-pole $1.5 \mathrm{~kW}$ IM. The IM is mechanically coupled to a magnetic clutch (MC), which provides rated load torque, even at very low speed. The main processor in Expert 3 control system is a floating point processor TMS320C6713 with a max clock speed of $225 \mathrm{MHz}$. All the algorithms including the TSRLS, the RLS algorithm, and some transformation modules are implemented in 


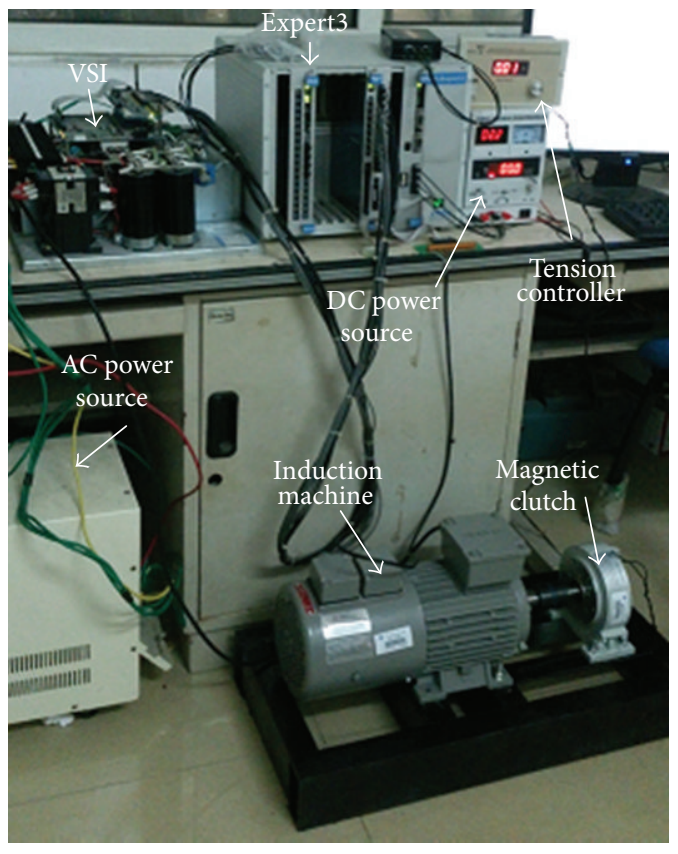

(a)

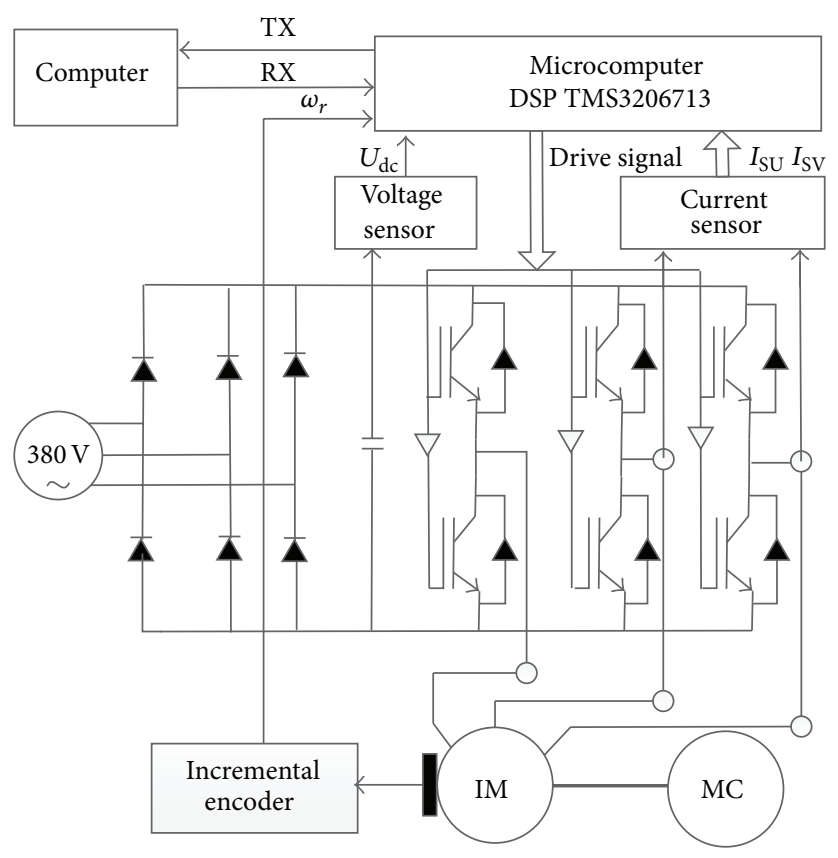

(b)

FIgURE 3: Complete drive system. (a) Picture of the experimental setup. (b) Functional block diagram of the experimental setup.

TABLE 1: Real electric parameters of the IM and the nameplate data.

\begin{tabular}{lc}
\hline Parameter & Value \\
\hline Nominal power & $1.5 \mathrm{~kW}$ \\
Nominal voltage & $380 \mathrm{~V}$ \\
Nominal current & $3.8 \mathrm{~A}$ \\
Nominal speed & $1450 \mathrm{rpm}$ \\
Pole pairs & 2 \\
Rotor resistance $R_{r}$ & $2.5 \Omega$ \\
Stator resistance $R_{s}$ & $3.6 \Omega$ \\
Stator inductance $L_{s}$ & $0.301 \mathrm{H}$ \\
Rotor inductance $L_{r}$ & $0.302 \mathrm{H}$ \\
Mutual inductance $L_{m}$ & $0.273 \mathrm{H}$ \\
\hline
\end{tabular}

TMS320C6713 with a $100 \mu$ s sampling time. The data acquisition of the parameter estimations, measured variable values, and their visualization are realized on the cockpit provided by PEView9 software. Insulated Gate Bipolar Transistor (IGBT) modules are driven by the PWM signal with a switching frequency of $10 \mathrm{kHz}$ and a dead time of $2 \mu \mathrm{s}$. The stator phase currents are measured via two hall-effect current sensors. The rotor angle and speed of the IM are measured from an incremental encoder with 2048 pulses per revolution.

(A) Experiment 1: Single-Phase AC Test. In this experiment, the reference $\beta$-axis component of the stator voltage $U_{s \beta}{ }^{*}$ is set to zero, whereas the $\alpha$-axis component $U_{s \alpha}{ }^{*}$ is generated from the current proportional $(P)$ regulator. As stated in [12], the injected waveform to induction machines must have at least two different harmonics for retrieving all the four
TABLE 2: Estimated electric parameters of the IM and the real ones.

\begin{tabular}{lcc}
\hline Parameter & Real & Estimated \\
\hline Stator resistance $R_{s}(\Omega)$ & 3.6 & 3.22 \\
Rotor resistance $R_{r}(\Omega)$ & 2.5 & 2.13 \\
Rotor inductance $L_{r}(\mathrm{H})$ & 0.302 & 0.272 \\
Stator inductance $L_{s}(\mathrm{H})$ & 0.301 & 0.26 \\
Mutual inductance $L_{m}(\mathrm{H})$ & 0.273 & 0.23 \\
Rotor time constant $T_{r}$ & 0.121 & 0.128 \\
\hline
\end{tabular}

$\theta\left(\theta_{1}, \theta_{2}, \theta_{3}, \theta_{4}\right)$ parameters, so the reference stator current $I_{s \alpha}{ }^{*}$ is chosen as $I_{s \alpha}{ }^{*}=1.5+1 \sin (157 t)+1.5 \sin (62.8 t)$ of which alternating component is composed of two sinusoids with different frequency at $10 \mathrm{~Hz}$ and $25 \mathrm{~Hz}$, respectively. The parameters of the first-order filter in measured signal vector $\varphi(k)$ are $h_{0}=40$ and $h_{1}=90$, and the $P$ regulator has the gain of $k_{p}=40$. The experimental results are shown in Figure 4. The actual stator current $I_{s \alpha}$ is slightly smaller than the reference stator current $I_{s \alpha}{ }^{*}$ in Figure 4(a) because only $P$ regulator is used in the current control loop. The oscillograms of $I_{s \alpha}, U_{s \alpha}{ }^{*}$, and $\left(d_{1}, d_{2}, d_{3}, d_{4}\right)$ are illustrated in Figures 4(b), 4(c), and 4(d) and they are good enough to ensure the accuracy of the TSRLS and RLS algorithm.

(B) Experiment 2: Parameter Estimation. The parametric vector $\theta$ and the electric parameters of the IM estimated by the RLS and TSRLS are shown in Figures 5 and 6 . Note that the difference in parameter estimations between the two estimation algorithms at steady state is tiny. The estimated electric parameters of the IM obtained by the TSRLS compared to the real ones are listed in Table 2 . The errors between 

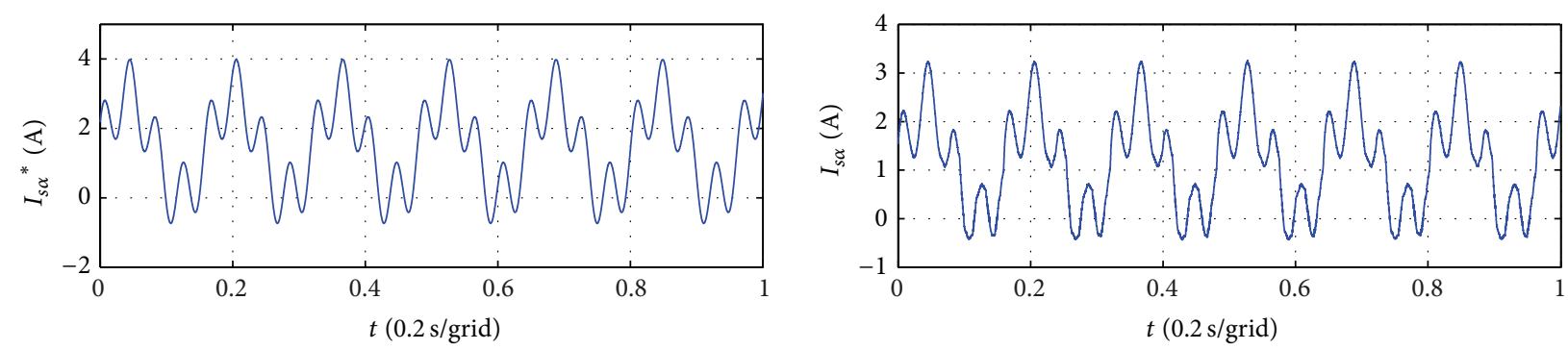

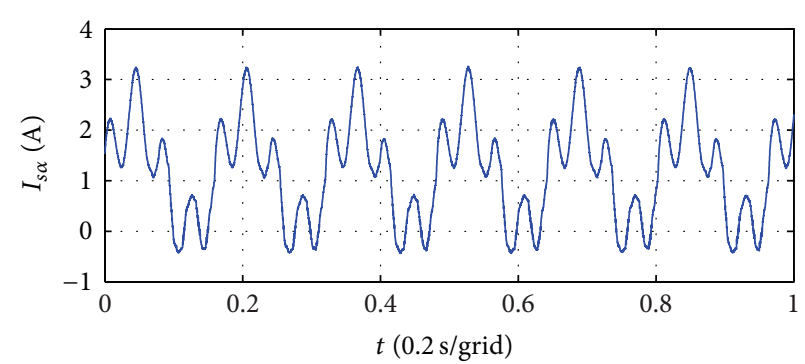

(a) Waveforms of $I_{s \alpha}{ }^{*}$ and $I_{s \alpha}$
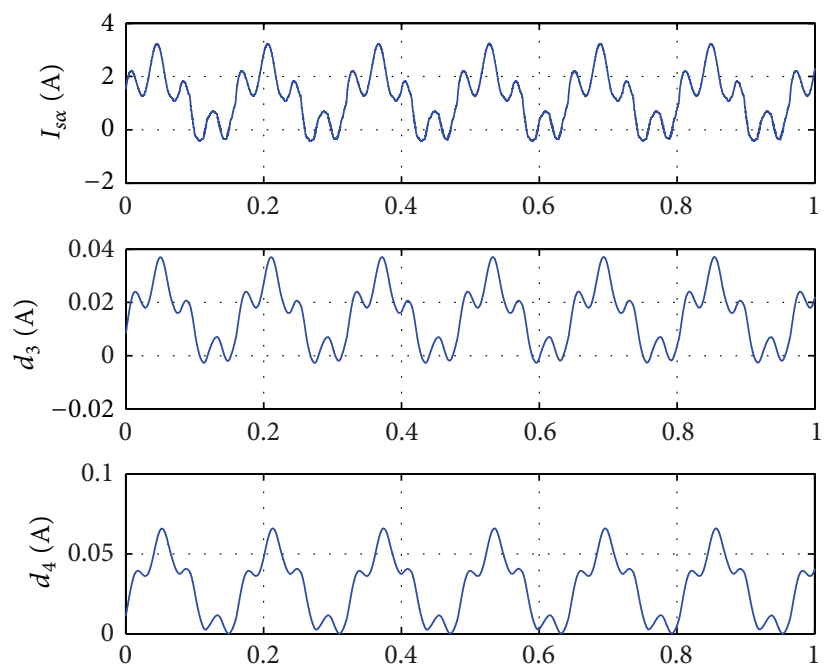

(c) Waveforms of $I_{s \alpha}, d_{3}$, and $d_{4}$

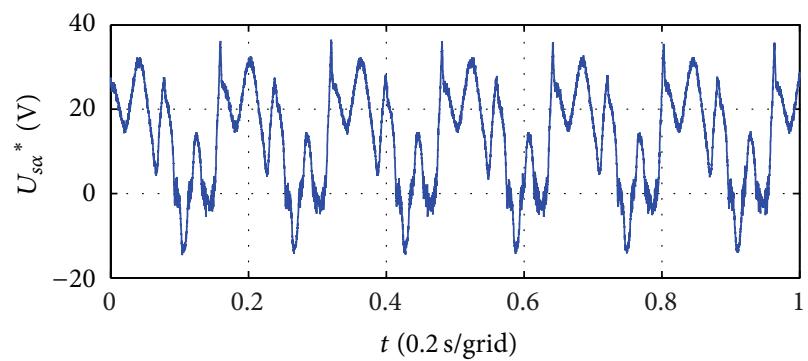

(b) Waveforms of $I_{s \alpha}$ and $U_{s \alpha}{ }^{*}$
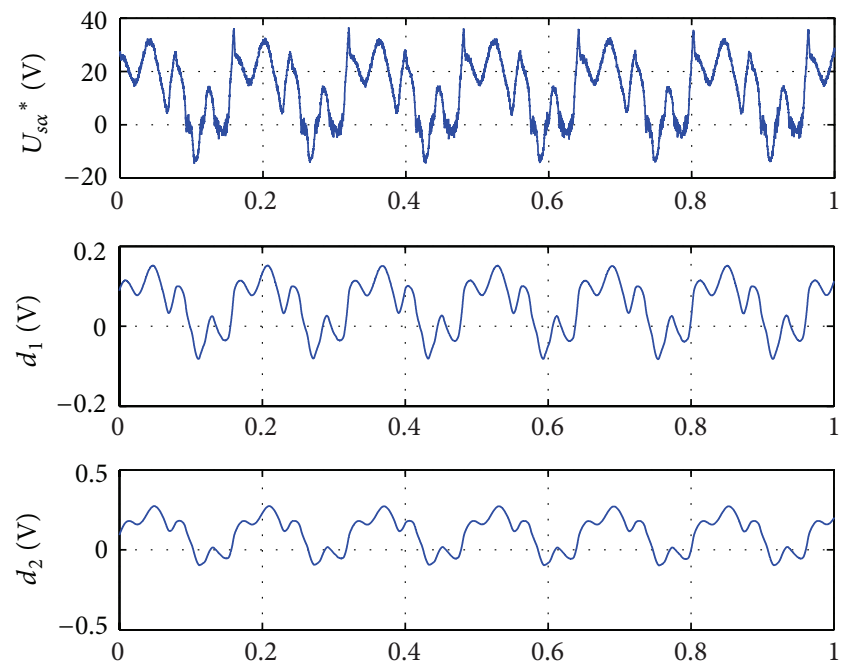

(d) Waveforms of $U_{s \alpha}{ }^{*}, d_{1}$, and $d_{2}$

FIgURE 4: Experimental waveforms.

real and estimated values are about $13 \%$, in which the highest $15.7 \%$ appears in the mutual inductance. The convergence time of these estimated electric parameters is less than $0.2 \mathrm{~s}$. The accuracy of the estimated electric parameters is sufficient for self-commissioning. The proposed method can also provide a good initialization of the machine parameters for online estimation techniques.

(C) Experiment 3: Online Verification for the Parameter Estimation. To further verify the accuracy of the proposed parameter estimation algorithm, the estimated parameter values are applied to the flux-oriented controlled IM. The estimated electric parameters of the IM are employed for the design of PI controllers and the extended Kalman filter (EKF) observer for rotor flux and speed estimation under speedsensorless condition. The performance of the FOC system of
IM depends on the accuracy of rotor flux observation and slip frequency calculation [18]. In this experiment, the reference rotor flux is set to $0.5 \mathrm{~Wb}$. The machine is accelerated from $0 \mathrm{rpm}$ to $600 \mathrm{rpm}$ at $0.4 \mathrm{~s}$ and the load torque is set to $2 \mathrm{Nm}$ at all times. The experimental results are gathered in Figure 7. Excellent speed-tracking performance is shown in Figures 7 (a) and 7(b) and the error between the real and estimated values is less than $5 \mathrm{rpm}$ at steady state (the error is more than $30 \mathrm{rpm}$ at standstill because the EKF observer is not fit for low speed identification [19]). The two current components $I_{s d}$ and $I_{s q}$ are shown in Figure 7(c). In Figure 7(d), the estimated rotor flux stabilizes at $0.5 \mathrm{~Wb}$, in accordance with the reference value. From this experiment, it is suggested that the estimated electric parameters of the IM are accurate enough for ensuring successful start-up and performing a practical field-oriented control. 


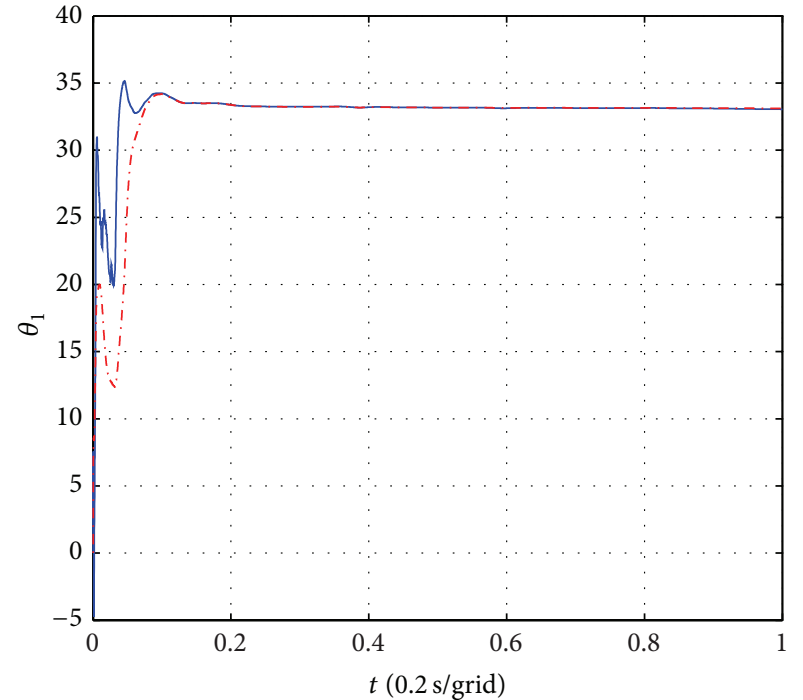

$\theta_{1}$ estimated (RLS)

... $\theta_{1}$ estimated (TSRLS)

(a) $\theta_{1}$ estimation

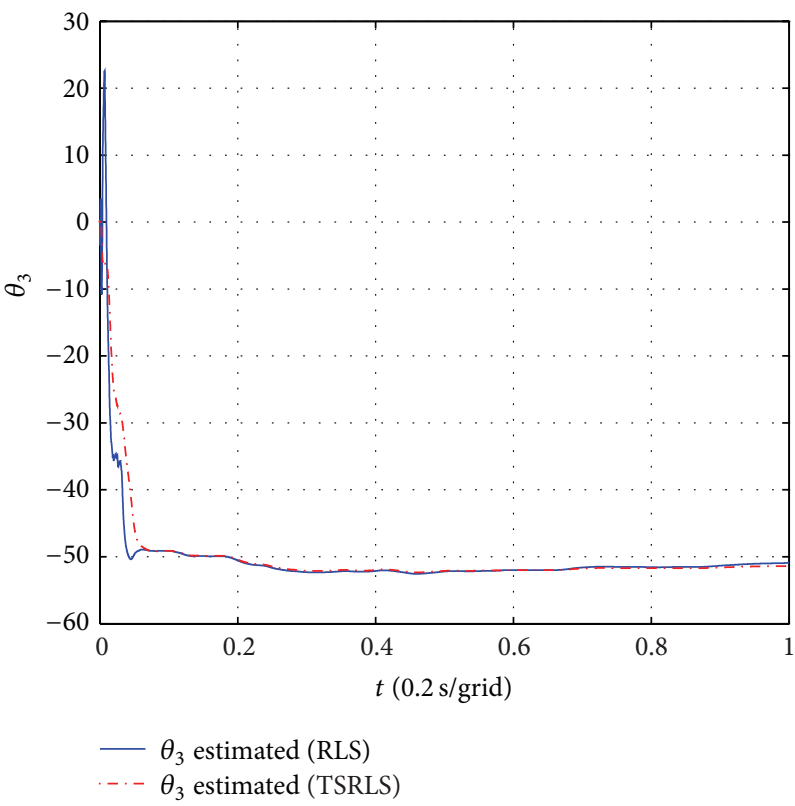

(c) $\theta_{3}$ estimation

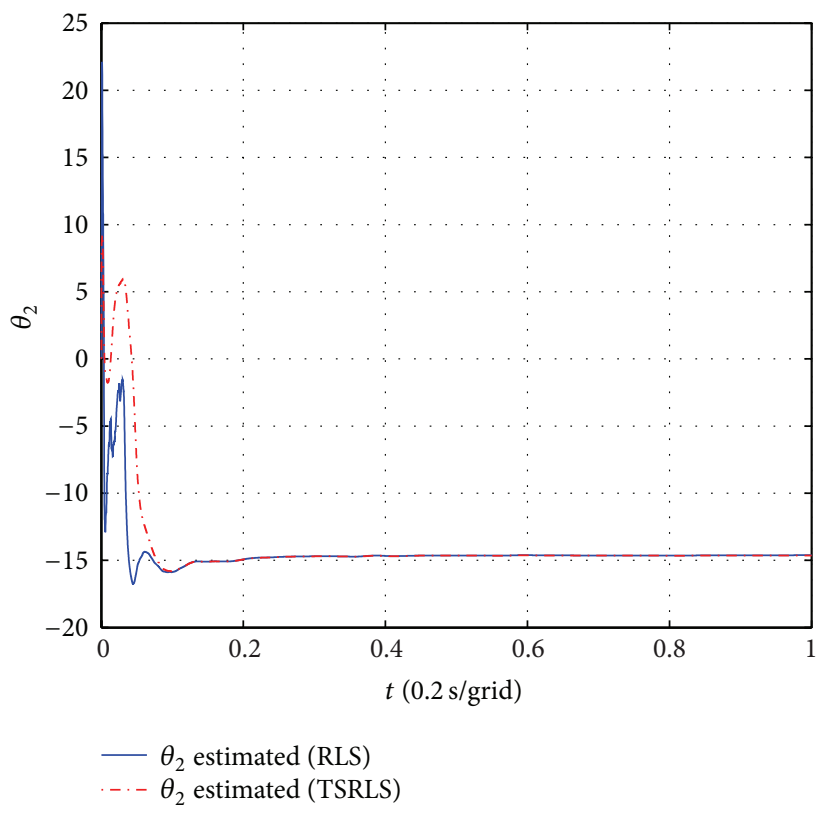

(b) $\theta_{2}$ estimation

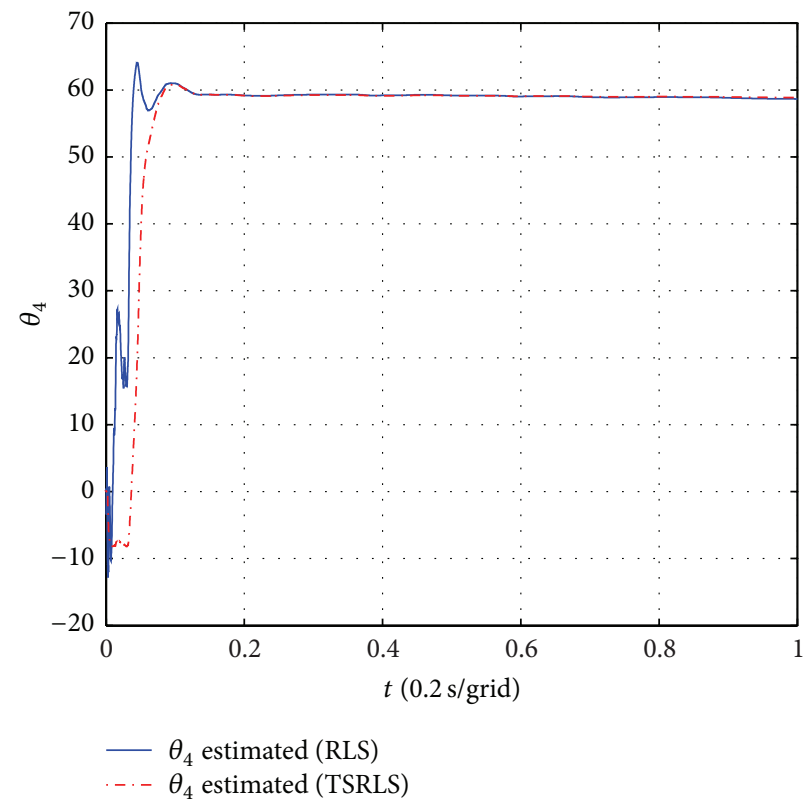

(d) $\theta_{4}$ estimation

FIGURE 5: Experimental results for parametric vector $\theta$ estimation (RLS and TSRLS).

(D) Numerical Complexity of the Algorithm. To illustrate the advantage of the proposed algorithm, the numbers of arithmetic operations required during each sample period by conventional RLS algorithm and the TSRLS are shown in Table 3, respectively. The dimensions of the state vectors $\varphi(t), \varphi_{a}(t)$, and $\varphi_{b}(t)$ are, respectively, $n, n_{a}$, and $n_{b}$. To perform conventional RLS algorithm in a sample period, 78 additions and 84 multiplications are calculated totally, whereas the number of additions and multiplications of the TSRLS algorithm in a sample period is 48 and 52, realizing a $38.5 \%$ decrease of the number of additions and a $38.5 \%$ decrease of the number of multiplications.

\section{Conclusion}

The real-time digital implementation of the conventional recursive least squares algorithm for electric parameter estimation of the IM at standstill requires a high-performance 


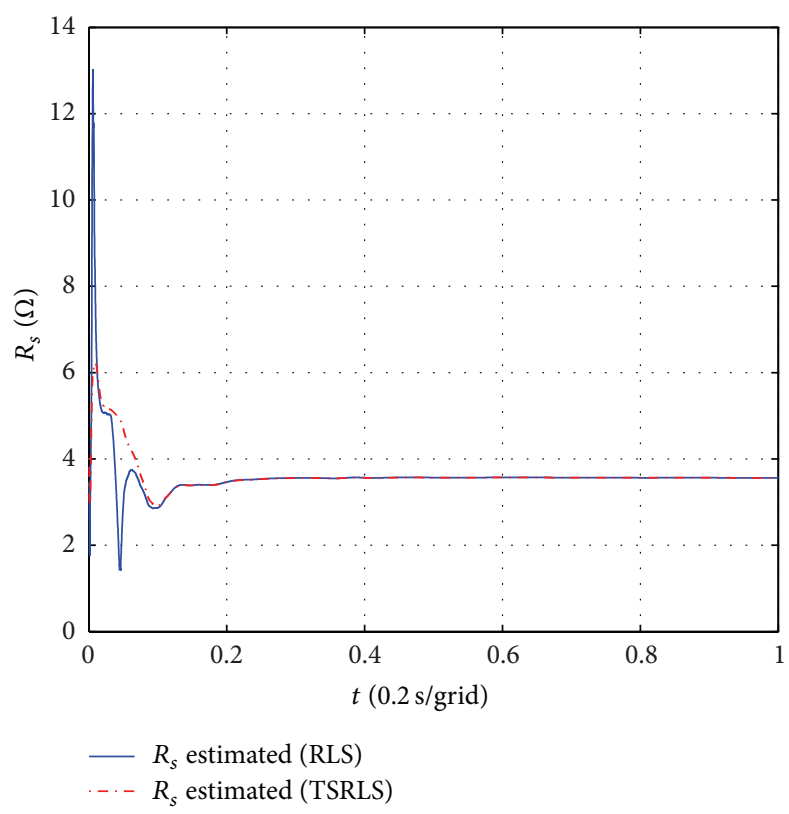

(a) Stator resistance estimation

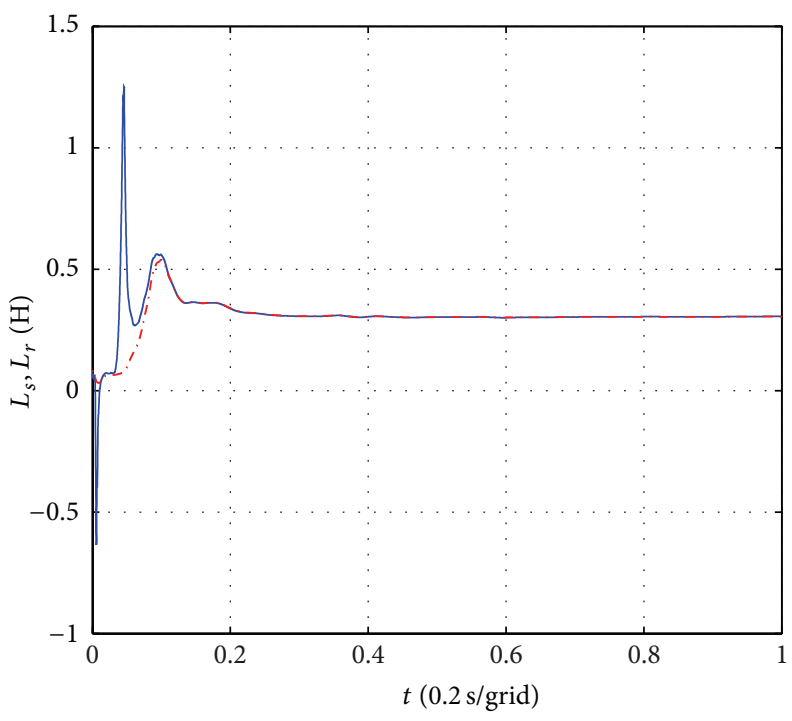

$-L_{s}, L_{r}$ estimated (RLS)

-..- $L_{s}, L_{r}$ estimated (TSRLS)

(c) Stator and rotor inductance estimation

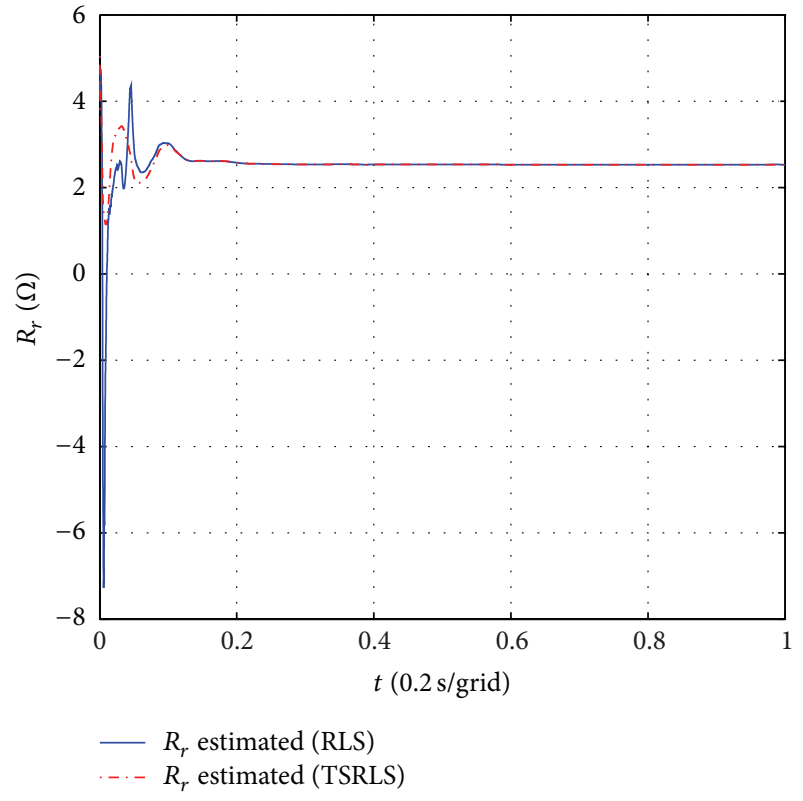

(b) Rotor resistance estimation

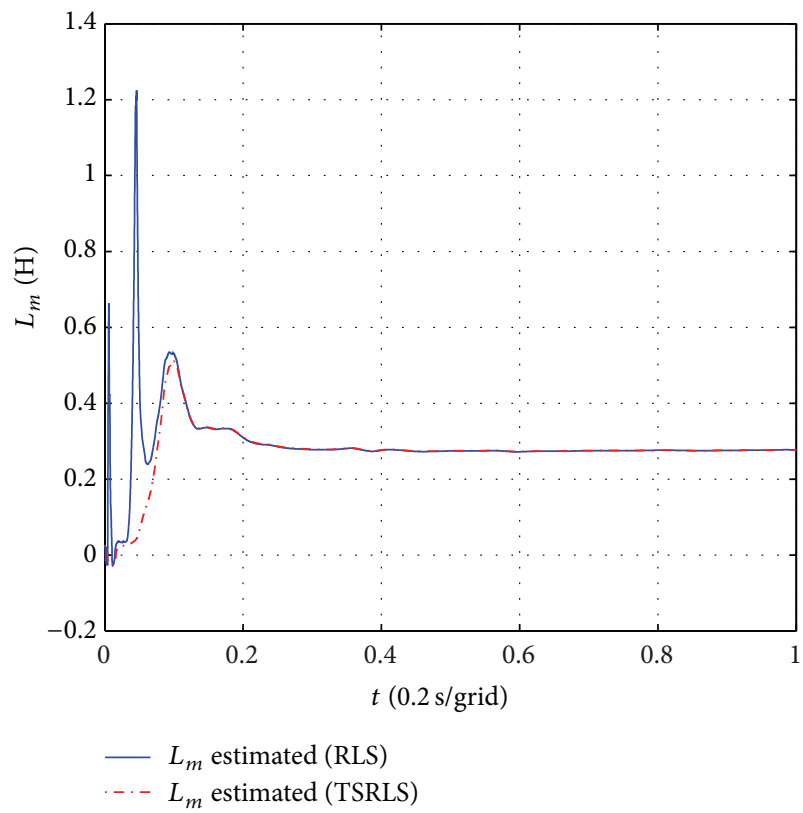

(d) Mutual inductance estimation

FIGURE 6: Experimental results for electric parameter estimation (RLS and TSRLS).

TABLE 3: RLS and TSRLS arithmetic operation requirements.

\begin{tabular}{lcc}
\hline Algorithm & $\begin{array}{c}\text { Number of } \\
\text { multiplications }\end{array}$ & Number of additions \\
\hline $\begin{array}{l}\text { RLS } \\
(n=4)\end{array}$ & $4 n^{2}+5 n(84)$ & $4 n^{2}+3 n+2(78)$ \\
$\begin{array}{l}\text { TSRLS } \\
\left(n_{a}=n_{b}=2\right)\end{array}$ & $4 n_{a}{ }^{2}+4 n_{b}{ }^{2}+5 n(52)$ & $4 n_{a}{ }^{2}+4 n_{b}{ }^{2}+3 n+4(48)$ \\
\hline
\end{tabular}

signal processor to perform complex mathematical calculations and manipulate a large amount of data. So this paper proposes the novel two-stage recursive least squares algorithm. The TSRLS can reduce computational cost effectively compared to the conventional RLS. The experimental results show that the TSRLS has good identifying performance. The TSRLS algorithm provides a new idea to solve computational complexity and allows using cheaper DSP in practical applications.

\section{Conflict of Interests}

The authors declare that there is no conflict of interests regarding the publication of this paper. 


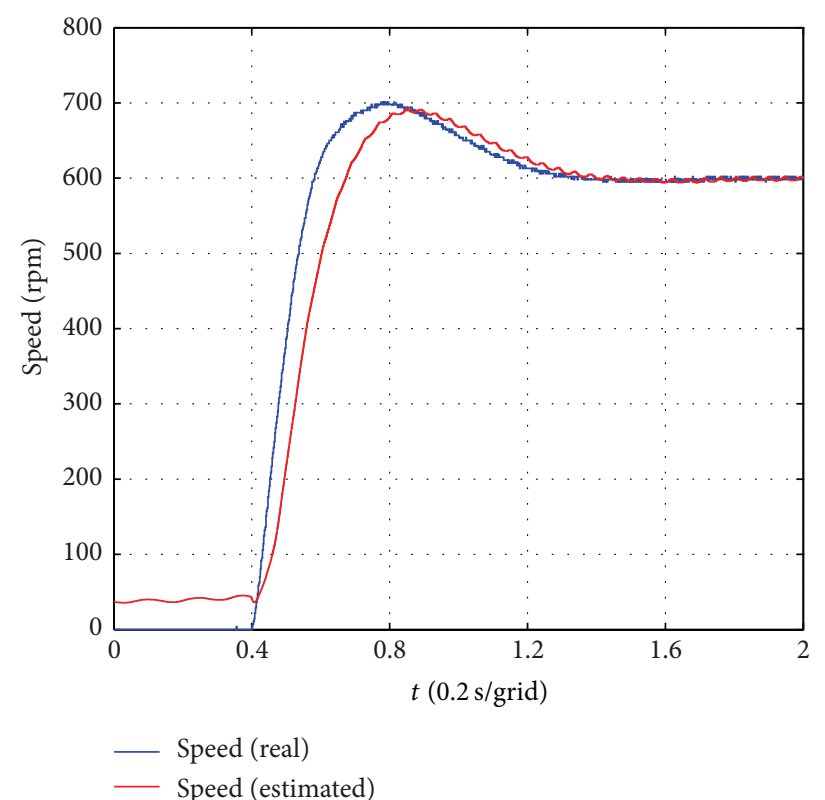

(a) Speed estimation

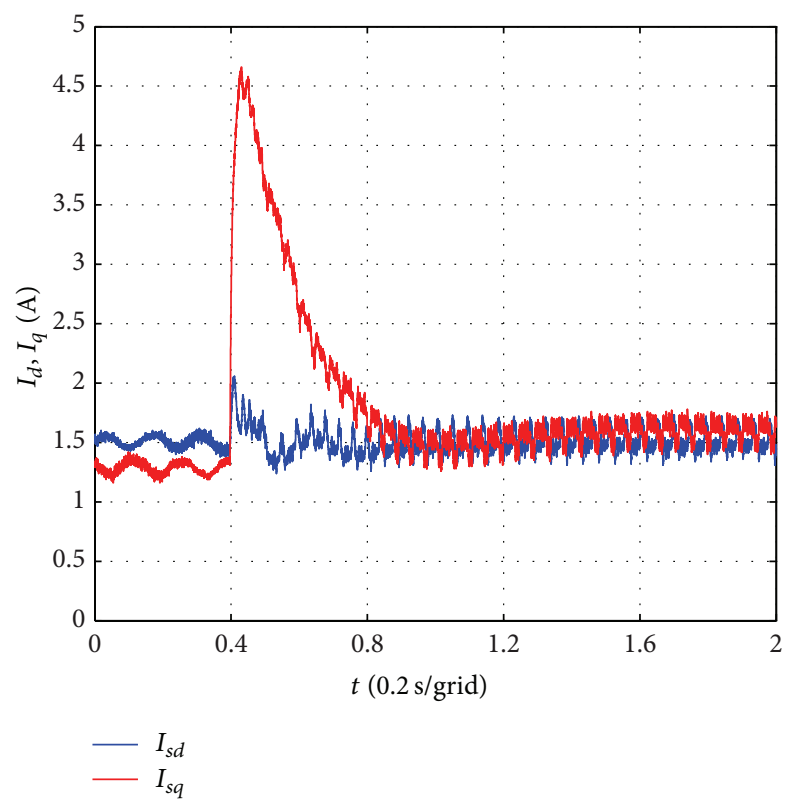

(c) $I_{s d}$ and $I_{s q}$

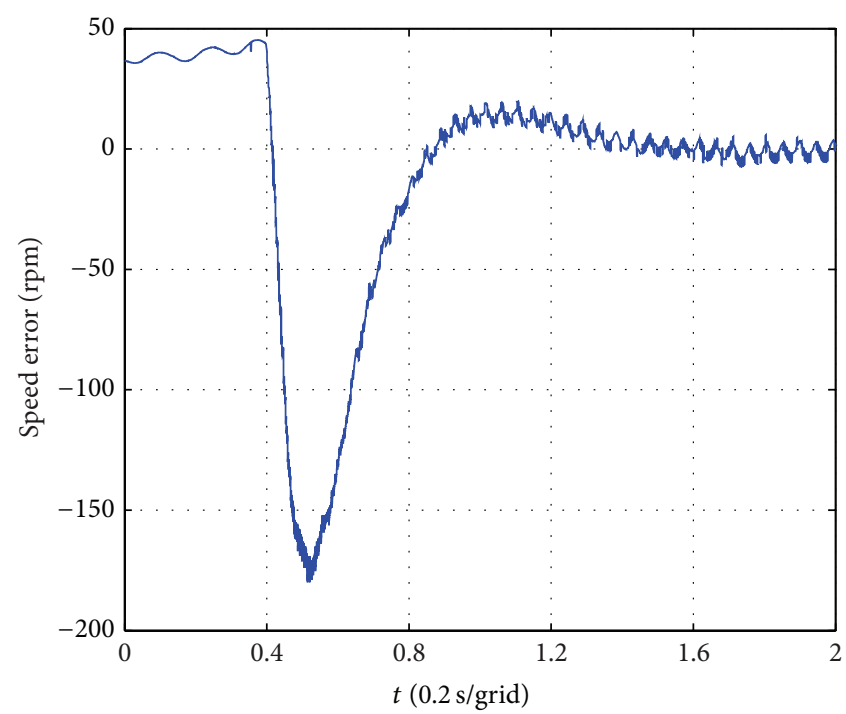

(b) Difference of speed estimation

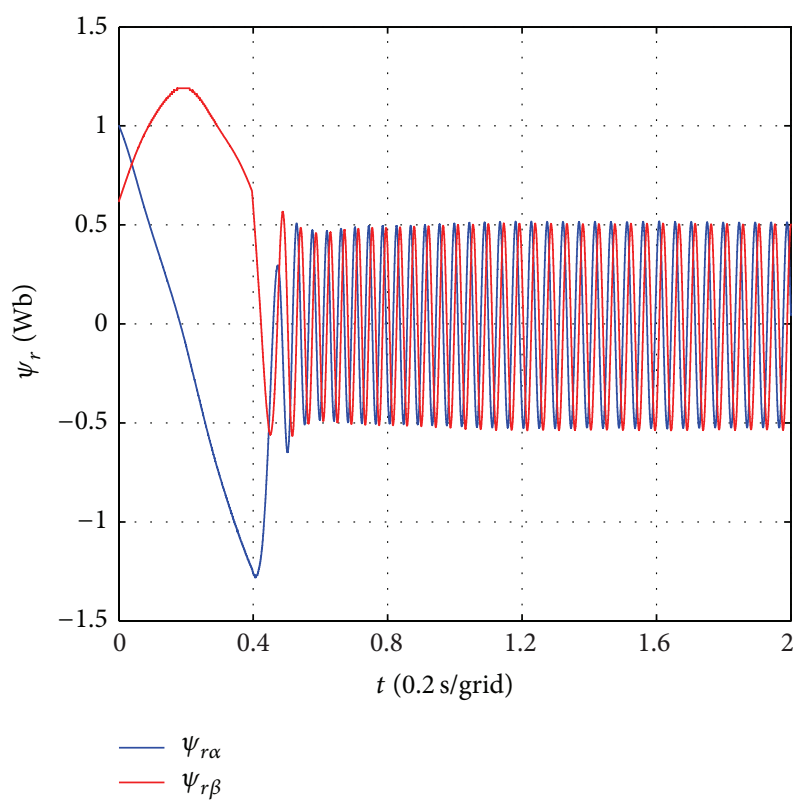

(d) Rotor flux estimation

Figure 7: Experimental results of online verification.

\section{References}

[1] Z. Zhang, R. Tang, B. Bai, and D. Xie, "Novel direct torque control based on space vector modulation with adaptive stator flux observer for induction motors," IEEE Transactions on Magnetics, vol. 46, no. 8, pp. 3133-3136, 2010.

[2] M. Farasat, A. M. Trzynadlowski, and M. S. Fadali, "Efficiency improved sensorless control scheme for electric vehicle induction motors," IET Electrical Systems in Transportation, vol. 4, no. 4, pp. 122-131, 2014.

[3] M. Barut, S. Bogosyan, and M. Gokasan, "Switching EKF technique for rotor and stator resistance estimation in speed sensorless control of IMs," Energy Conversion and Management, vol. 48, no. 12, pp. 3120-3134, 2007.

[4] F. R. Salmasi and T. A. Najafabadi, "An adaptive observer with online rotor and stator resistance estimation for induction motors with one phase current sensor," IEEE Transactions on Energy Conversion, vol. 26, no. 3, pp. 959-966, 2011.

[5] Y. He, Y. Wang, Y. Feng, and Z. Wang, "Parameter identification of an induction machine at standstill using the vector constructing method," IEEE Transactions on Power Electronics, vol. 27, no. 2, pp. 905-915, 2012. 
[6] P. Castaldi and A. Tilli, "Parameter estimation of induction motor at standstill with magnetic flux monitoring," IEEE Transactions on Control Systems Technology, vol. 13, no. 3, pp. 386400, 2005.

[7] M. O. Sonnaillon, G. Bisheimer, C. De Angelo, and G. O. García, "Automatic induction machine parameters measurement using standstill frequency-domain tests," IET Electric Power Applications, vol. 1, no. 5, pp. 833-838, 2007.

[8] Y.-S. Kwon, J.-H. Lee, S.-H. Moon, B.-K. Kwon, C.-H. Choi, and J.-K. Seok, "Standstill parameter identification of vector controlled induction motors using the frequency characteristics of rotor bars," IEEE Transactions on Industry Applications, vol. 45, no. 5, pp. 1610-1618, 2009.

[9] M. Carraro and M. Zigliotto, "Automatic Parameter identification of inverter-fed induction motors at standstill," IEEE Transactions on Industrial Electronics, vol. 61, no. 9, pp. 46054613, 2014.

[10] L. Peretti and M. Zigliotto, "Automatic procedure for induction motor parameter estimation at standstill," IET Electric Power Applications, vol. 6, no. 4, pp. 214-224, 2012.

[11] S. J. Underwood and I. Husain, "Online parameter estimation and adaptive control of permanent-magnet synchronous machines," IEEE Transactions on Industrial Electronics, vol. 57, no. 7, pp. 2435-2443, 2010.

[12] M. Cirrincione, M. Pucci, and G. Vitale, "A least-squares based methodology for estimating the electrical parameters of induction machine at standstill," in Proceedings of the IEEE International Symposium on Industrial Electronics (ISIE '02), pp. 541-547, July 2002.

[13] S. J. Underwood and I. Husain, "Online parameter estimation and adaptive control of permanent-magnet synchronous machines," IEEE Transactions on Industrial Electronics, vol. 57, no. 7, pp. 2435-2443, 2010.

[14] A. J. Netto, P. R. Barros, C. B. Jacobina, and A. M. N. Lima, "Estimating the parameters of an induction motor in openloop and closed-loop operation," in Proceedings of the 39th IAS Annual Meeting. Conference Record of the IEEE Industry Applications Conference, vol. 1, pp. 26-31, IEEE, October 2004.

[15] C.-H. Fang, S.-K. Lin, and S.-J. Wang, "On-line parameter estimator of an induction motor at standstill," in Proceedings of the European Control Conference (ECC '03), vol. 1, pp. 32003203, Cambridge, UK, September 2003.

[16] N. A. A. Shashoa, A. S. Emhemmed, and S. K. Yakhlef, "Fault detection based on two-stage recursive least squares parameter estimation," in Proceedings of the 14th International Conference on Sciences and Techniques of Automatic Control and Computer Engineering (STA '13), pp. 382-387, Sousse, Tunisia, December 2013.

[17] H. Duan, J. Jia, and R. Ding, "Two-stage recursive least squares parameter estimation algorithm for output error models," Mathematical and Computer Modelling, vol. 55, no. 3-4, pp. 11511159, 2012.

[18] D. Casadei, F. Profumo, G. Serra, and A. Tani, "FOC and DTC: two viable schemes for induction motors torque control," IEEE Transactions on Power Electronics, vol. 17, no. 5, pp. 779-787, 2002.

[19] F. Alonge, F. D’Ippolito, and A. Sferlazza, "Sensorless control of induction-motor drive based on robust kalman filter and adaptive speed estimation," IEEE Transactions on Industrial Electronics, vol. 61, no. 3, pp. 1444-1453, 2014. 


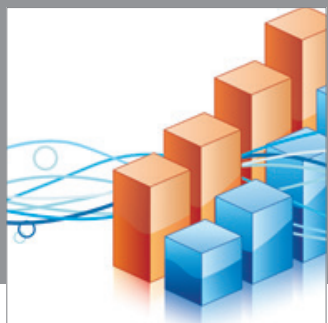

Advances in

Operations Research

mansans

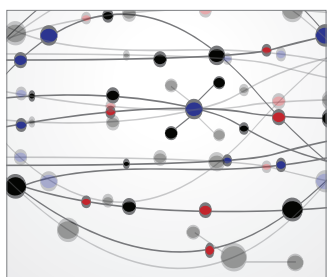

The Scientific World Journal
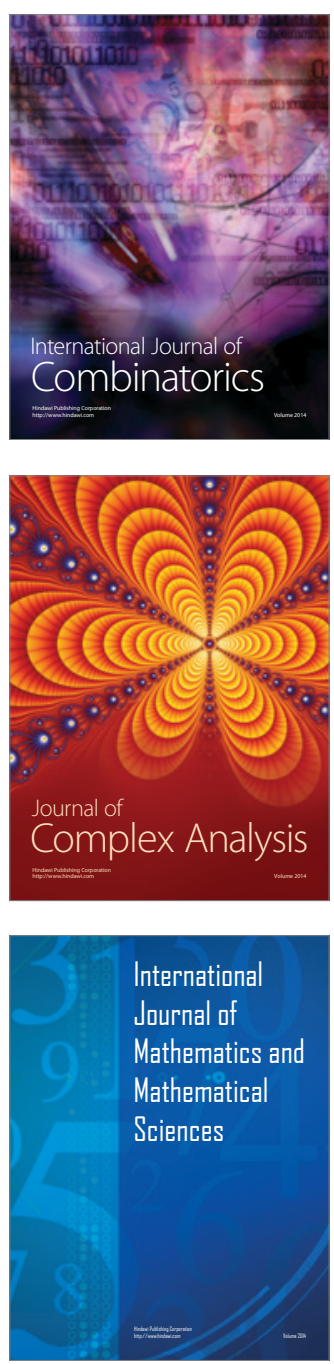
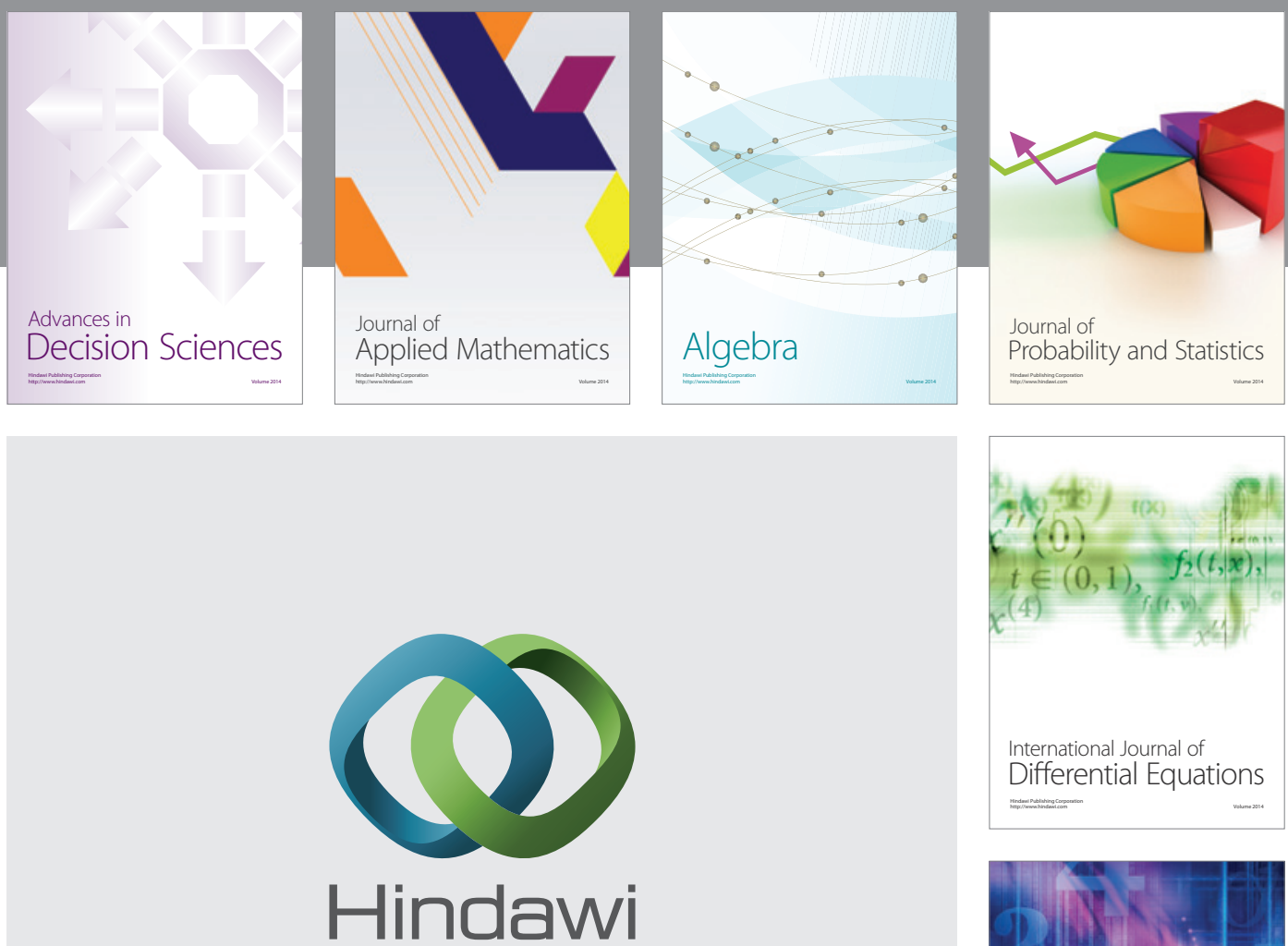

Submit your manuscripts at http://www.hindawi.com
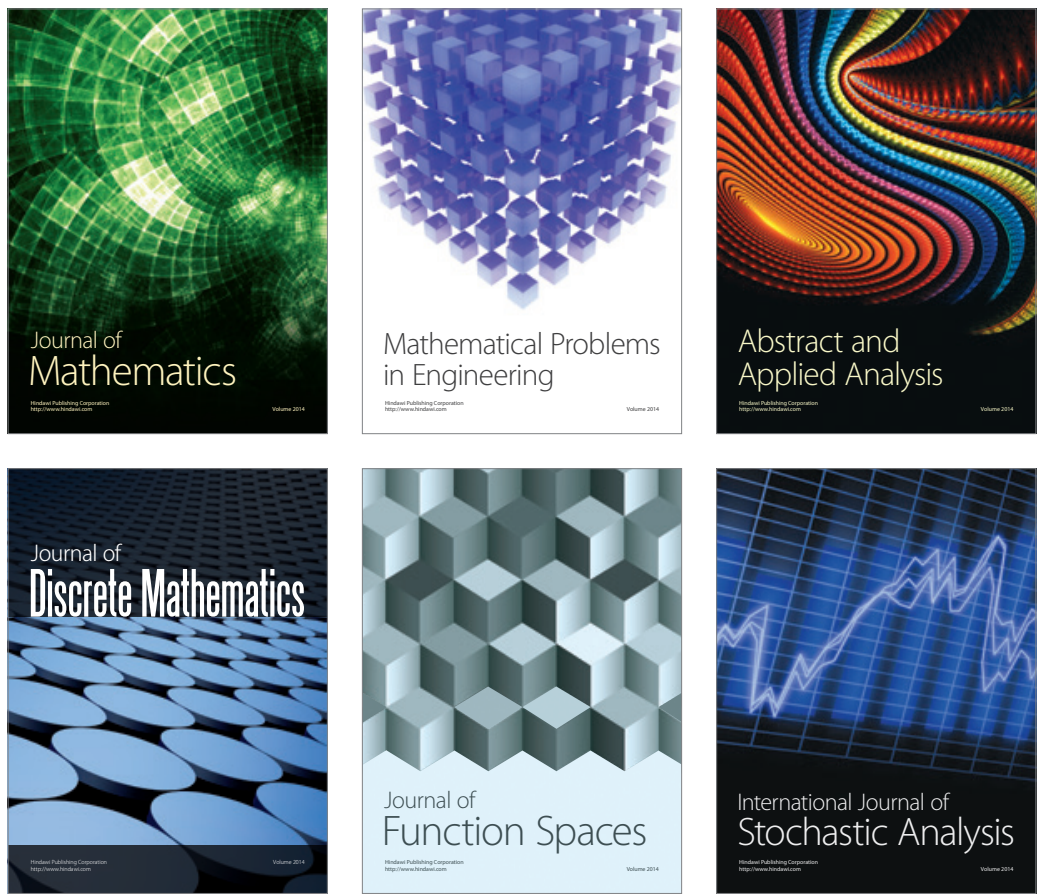

Journal of

Function Spaces

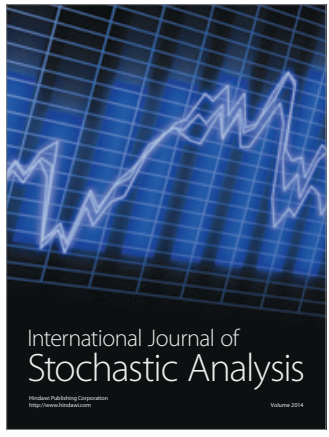

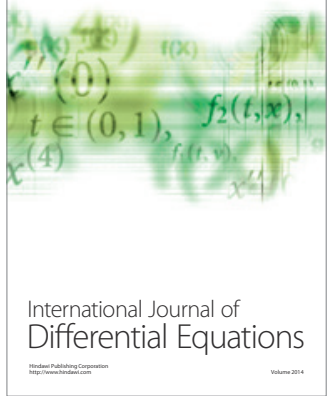
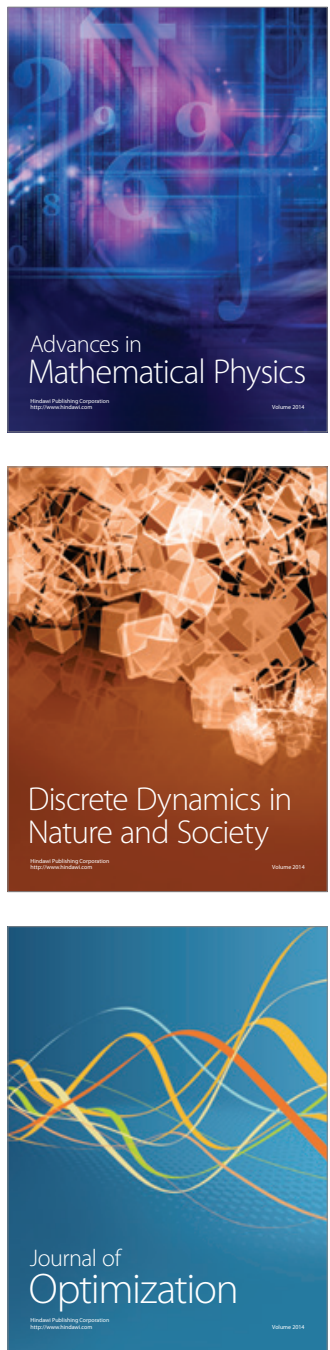